\title{
Die Angst des Ministeriums vor der Aufsicht - \\ Das Bundesfinanzministerium in der deutschen Finanzmarktpolitik
}

\author{
Stefan Handke
}

\section{Ministerial Weaknesses in German Financial Market Supervision}

Abstract: The financial crisis revealed weaknesses in the policy making and supervisory capacities of the German Ministry of Finance (BMF). Due to a lack of adequate personnel and the challenges of unexpected tasks, the ministry had to outsource certain functions in policy formulation. However, it did not only mandate the German Supervisory Authority (BaFin), but also law firms like Freshfields. As a matter of fact, the crisis showed only the tip of the iceberg, because the BMF is not even able to cope with its responsibilities in daily business. Institutional reforms, which were discussed in German politics, did not deal with this problem, but settled for symbolic actions.

Keywords: Financial Market Policy, Ministry of Finance, BaFin, Delegation Problems

Schlagwörter: Finanzmarktpolitik, Finanzministerium, BaFin, Steuerungsprobleme

\section{Einleitung}

Wenige Jahre nach dem Höhepunkt der Finanzmarktkrise haben sich in Deutschland weder das Wirtschaftssystem noch die staatliche Finanzaufsicht grundlegend verändert. Dabei hatte die Krise einerseits wirtschaftspolitische Grundsatzfragen und Fragen der Wirtschaftsethik auf die politische Tagesordnung gebracht, andererseits aber auch die Überprüfung der Finanzmarktregulierung und -aufsicht, also die Schaffung von Vorschriften und die Durchsetzung von Regeln im Finanzsektor durch staatliche Institutionen (vgl. Handke 2010a). In diesem Zusammenhang sollte das System der Finanzmarktaufsicht mit der Bundesanstalt für Finanzdienstleistungsaufsicht (BaFin), der Deutschen Bundesbank und dem Bundesministerium der Finanzen (BMF) reformiert werden. Die BaFin, die nach dem Finanzdienstleistungsaufsichtsgesetz (FinDAG) als Allfinanzaufsichtsbehörde für die Markt- und Solvenzaufsicht über Finanzunternehmen zuständig ist, war wegen der Verwerfungen im Finanzsektor unter Druck geraten. Gleichzeitig verlor sie den politischen Rückhalt der Regierung, da die Behördenleitung durch medienwirksame Verlautbarungen und nicht abgestimmte Alleingänge für öffentliche Unruhe im Finanzmarkt sorgte (Handelsblatt 18.09.2007, 26.10.2009). Heftiger Kritik sah sich insbesondere Jochen Sanio als Präsident der BaFin ausgesetzt, der zwar fachlich angesehen ist, jedoch häufig undiplomatisch agierte und damit sein eigenes Ansehen und das der BaFin gefährdete.

Neben der BaFin sind wegen der besonderen Betroffenheit von Banken in der Finanzkrise auch die Deutsche Bundesbank, die für Bankenprüfungen zuständig ist, und das BMF in den Blick des öffentlichen Interesses gerückt. Während die 
Kritik an der Bundesbank mit Verweis auf ihre Unabhängigkeit und ihre gute Arbeit als Notenbank schnell verstummte, sah sich das BMF einer etwas längeren Debatte um seine Verantwortlichkeit ausgesetzt (vgl. u. a. Hulverscheidt 2008; Weiland u. Goetz 2009). Dies hat unter anderem zur Folge, dass das Ministerium auch drei Jahre nach Ausbruch der Krise mit äußerster Zurückhaltung auf Nachfragen zu seiner Arbeit in der Finanzaufsicht reagiert (vgl. Interviewnachweis 1) ${ }^{1}$. Der Fall der Hypo Real Estate Bank (HRE), die mit staatlichen Mitteln vor dem Zusammenbruch gerettet werden musste, hatte jedoch die Einsetzung eines Untersuchungsausschusses des Deutschen Bundestages zur Folge, um Aufsichtsversäumnisse im Vorfeld und während der Krise zu prüfen. Bei den Anhörungen im Ausschuss zeigten sich neben kleineren Problemen in der Zusammenarbeit von Bundesbank und BaFin vor allem Schwächen in der Arbeit des Finanzministeriums. Dieses konnte weder seine Regulierungs- noch seine Aufsichtszuständigkeiten angemessen ausführen und musste für zentrale ,Krisengesetze ' die Politikformulierung an Rechtsanwaltskanzleien auslagern (vgl. Deutscher Bundestag 2009). Bei der Kontrolle der Finanzmarktaufsicht war das Ministerium beinahe vollständig von BaFin und Bundesbank abhängig und konnte deren Berichte nicht in ausreichendem Umfang prüfen (vgl. Deutscher Bundestag 2009a). Diese Mängel der ministeriellen Performanz der vergangenen Jahre scheinen dabei nicht allein der Schwere der Krise geschuldet zu sein, sondern einem Defizit in der Ressourcenausstattung des BMF.

Dieser Beitrag widmet sich daher der grundsätzlichen Frage nach der regulatorischen und aufsichtlichen Leistungsfähigkeit des Finanzministeriums. Dazu gehören neben Aspekten der Finanzmarktpolitik auch die Bereiche der ministeriellen Kontroll- und Steuerungsfähigkeit gegenüber der BaFin als nachgeordneter Behörde im Geschäftsbereich des BMF. Sowohl für die Finanzmarktpolitik als auch für die Steuerung der BaFin wird von ministeriellen Unzulänglichkeiten in personeller, struktureller und kapazitärer Hinsicht ausgegangen, die zu einer Angst des Ministeriums vor der Aufsicht führen. Bei Organisationen findet sich freilich keine Angst als affektiver Zustand im individualpsychologischen Sinne. Vielmehr soll durch die Verwendung des Begriffs eine durch das Ministerium selbst wahrgenommene Schwäche im organisatorischen Lernen, in der Reaktionsfähigkeit auf Probleme und in der Erreichung eines zugeschriebenen Leistungsniveaus angedeutet werden (vgl. Baecker 2003). Die plakative, rhetorische Zuspitzung hebt einerseits auf den Bereich der funktionalen Aufsicht, also die Beaufsichtigung und Regulierung des Finanzsektors, und andererseits auf die Rechts- und Fachaufsicht als ministerielle Wahrnehmung der administrativen Aufsicht über die BaFin ab.

Zum einen ist das BMF als zuständige oberste Bundesbehörde nicht in der Lage die angemessene bzw. ausreichende Regulierung und Beaufsichtigung des deutschen Finanzmarktes sicherzustellen. Damit betreffen die Unzulänglichkeiten des Ministeriums den Aufgabenbereich der Regulierung, der die Vorschriften des

1 Der Autor führte von Juni bis Oktober 2010 Interviews mit Experten aus Politik, Verwaltung und Industrie. In allen Fällen war eine anonyme Behandlung der Aussagen die Voraussetzung für die Interviewteilnahme, sodass Interviews nur nummeriert angegeben werden können, ohne einen direkten Bezug zwischen Aussage und Quelle herzustellen. 
deutschen Banken-, Versicherungs- und Wertpapiersektors schaffen soll, ebenso wie die Implementation dieser Regelungen durch aufsichtliches Handeln. Für Regulierung und Aufsicht ist das BMF zwar zuständig, muss sich jedoch auf die Arbeit anderer staatlicher Akteure wie BaFin und Bundesbank oder sogar privater Vertragspartner stützen.

Zum anderen besteht die Gefahr eines Steuerungsverlustes gegenüber der Aufsichtsbehörde BaFin, die den größten Teil der Aufgaben erfüllt, für die das BMF verantwortlich ist. Die BaFin unterliegt dabei als nachgeordnete Behörde der Rechts- und Fachaufsicht des Ministeriums. Dies ist einerseits aufgrund der gewerbepolizeilichen Eingriffsrechte der BaFin im Markt und andererseits wegen der politischen Letztverantwortlichkeit des BMF zu erklären. Die Ausübung der Fachaufsicht stößt allerdings an Grenzen, da die ministeriellen Zugriffsmöglichkeiten auf die BaFin durch den Anstaltsstatus, der unter anderem eigene Budgetund Organisationsrechte einräumt, eingeschränkt sind. Darüber hinaus ist aufgrund begrenzter personeller Ressourcen auch eine inhaltliche Weisungskompetenz allenfalls selektiv wahrnehmbar. Dennoch durchbricht die Unterstellung der BaFin unter die ministerielle Fachaufsicht die traditionelle Typologie deutscher Behörden. Die BaFin wurde per Gesetz als Anstalt des öffentlichen Rechts gegründet, also in einer Rechtsform, die üblicherweise weisungsfrei ist (vgl. Bach et al. 2010). Das Delegationsverhältnis zwischen BMF und BaFin ist daher ein Sonderfall in der deutschen Verwaltung, der besondere Beachtung verdient, wenn die Performanz des Finanzministeriums infrage gestellt wird, da ein Spannungsverhältnis zwischen politisch intendierter Autonomie der BaFin und ministeriellen Steuerungsinteressen besteht (vgl. Frach 2008).

Das BMF beschreibt in einer Publikation zur Selbstdarstellung, dass ein „stabiles Finanzsystem [...] eine zentrale Rolle für die wirtschaftliche Entwicklung in Deutschland [spielt]“, und dass die ministerielle Aufgabe darin bestehe, „unter anderem die gesetzlichen Rahmenbedingungen [zu erarbeiten], damit die Finanzmärkte ihre volkswirtschaftliche Funktion erfüllen können“, wozu auch gehöre, die „Reformen der Finanzmarktregulierung und Finanzaufsicht auf nationaler, europäischer und weltweiter Ebene voranzutreiben“ (BMF 2010). Das BMF verfolgt damit hoch gesteckte Ziele, die es möglicherweise nicht erfüllen kann, weil sich Defizite bei der Programmformulierungs- und Behördensteuerungskapazität bemerkbar machen. Diese Annahme zu überprüfen und damit die Frage nach der Leistungsfähigkeit des Finanzministeriums zu beantworten, ist der Anspruch dieses Beitrages, der zunächst das BMF als politikfeldspezifischen Akteur darstellt, der behördliche Eigeninteressen verfolgt. Dabei umfassen strategische Überlegungen nicht nur den inhaltlichen Politikbereich (policy), sondern auch die Positionierung der Behörde im Politikfeld (politics) und - in Anlehnung an den RationalChoice-Institutionalismus - das antizipierte Verhalten anderer Akteure (vgl. Hall u. Taylor 1996). Daran anknüpfend wird die Delegationsbeziehung zwischen BMF und BaFin untersucht, die sich durch besondere Prinzipal-Agenten-Probleme auszeichnet. In diesem Zusammenhang findet auch das Auseinanderfallen von ministerieller Verantwortlichkeit und aufsichtsbehördlicher Verantwortung Beachtung, das sich aufgrund der Arbeitsteilung zwischen BMF und BaFin einstellt. Abschließend werden die Reformdiskussionen zur deutschen Finanzaufsicht im 
Lichte der gewonnenen Erkenntnisse bewertet, um beurteilen zu können, ob diese Maßnahmen, die ab 2009 diskutiert wurden, einen Beitrag zur Erreichung der selbstgesetzten Ziele des BMF leisten könnten. Die getroffenen Aussagen stützen sich dabei nicht nur auf öffentlich zugängliche Quellen, sondern auch auf eigene Erhebungen. Im Jahr 2010 wurden neun jeweils ein- bis zweistündige Interviews mit Vertretern von Aufsicht, Politik und Industrie geführt, in denen Hintergrundinformationen und Einschätzungen zur Finanzmarktkrise und den politikfeldspezifischen Reaktionen anhand eines Leitfadens gewonnen wurden. Als besonders gering erwies sich hierbei die Mitwirkungs- und Auskunftsbereitschaft staatlicher Akteure, insbesondere des BMF, das bis 2011 jede Form von Interview oder sonstiger Mitwirkung an Forschungsarbeiten verweigerte. Die Beobachtungen und Schlussfolgerungen dieses Beitrages basieren daher vorwiegend auf Informationen aus dem direkten Umfeld des Ministeriums und auf beantworteten Anfragen nach dem Informationsfreiheitsgesetz.

\section{Das Finanzministerium im Politikfeld Finanzmarktpolitik}

Das Bundesministerium der Finanzen ist die zentrale Behörde in der Finanzmarktpolitik, von der ausgehend sich Kompetenzen, Interessen und Beziehungen anderer Akteure ableiten und erklären lassen. Ein Charakteristikum der Finanzmarktpolitik ist die staatliche Regulierung des Geschäftsbetriebs von Banken, Versicherungen und Wertpapierhändlern, die stärker ausgeprägt ist als in anderen Politikfeldern (vgl. Frach 2010). Hier kann zunächst die Unterscheidung in Markt- und Risikoregulierung vorgenommen werden, um zu verdeutlichen, welche Bereiche des Politikfeldes regulierungsbedürftig sind (Döhler 2006, S. 208). Marktregulierung umfasst alle staatlichen Maßnahmen, die darauf gerichtet sind, einen funktionierenden Marktwettbewerb herzustellen oder aufrechtzuerhalten, so zum Beispiel das Verbot von illegalen Kartellen, die Trennung von Stromnetz und Stromerzeugung im Energiesektor oder die Freigabe von ehemals monopolistischen Telekommunikationsnetzen für Wettbewerber. In den Bereich der Risikoregulierung fallen hingegen Maßnahmen, die ökonomischen Wettbewerb nicht herstellen und fördern sollen, sondern diesem einen Rahmen geben, um Verbraucher vor schädigenden Auswirkungen der Produktion oder des Vertriebs von Produkten und Dienstleistungen zu schützen. Zur Risikoregulierung zählen unter anderem Arzneizulassungsbestimmungen, Vorgaben für Produktstandards oder Hygiene- und Fütterungsstandards in der Nutztierhaltung.

In der deutschen Finanzmarktpolitik ist der Verbraucherschutz eine bedeutende Herausforderung für staatliches Handeln, da sich der Finanzmarkt durch ausgeprägte Informationsasymmetrien auszeichnet, die mehrheitlich zu Lasten der Verbraucher gehen (vgl. Barth 2004). Diesen ist in der Regel nicht bekannt, in welcher geschäftlichen Situation sich eine Bank oder eine Versicherung befindet, bei der sie Geld anlegen oder Risiken absichern. Selbst wenn die Informationen darüber grundsätzlich existieren, fehlt ihnen häufig das Wissen, um diese zu verarbeiten (vgl. Kessler 2008, S. 19). Das bestehende Problem der "hidden information“ 
wird daher durch staatliche Regulierung so behoben, dass mündige Verbraucher ${ }^{2}$ in die Lage versetzt werden, Finanzprodukte sowie deren Risiko selbst ausreichend beurteilen und eine entsprechende Vertragsentscheidung treffen zu können. In Fällen, in denen Verbraucher solche Beurteilungen nicht vornehmen können, erfüllt die staatliche Aufsicht diese Aufgabe und schafft damit einerseits ersatzweise eine Vertrauensbasis für das Funktionieren des Marktes, andererseits schützt sie Verbraucher vor bestimmten Risiken. Neben diesem Verbraucherschutz verfolgt die Risikoregulierung den Schutz des Finanzsystems in seiner Gesamtheit. Dies umfasst ein Schutzziel für die Allgemeinheit, das Kunden, Finanzunternehmen und indirekt betroffene Dritte einbezieht.

An der Regulierung und Aufsicht in der Finanzmarktpolitik sind neben BaFin, Deutscher Bundesbank und BMF auch Landesbehörden beteiligt. Letztere werden jedoch in den weiteren Ausführungen ebenso außer Acht gelassen wie andere Politikfeldakteure, zu denen vor allem Verbraucher, Interessengruppen und Branchenverbände gehören. Sie alle spielen zwar für Entscheidungen und Prozesse im Politikfeld eine Rolle, haben aber für die hier gewählte Betrachtung des BMF nur geringe Bedeutung. Zentral sind hingegen die politischen Akteure, die Bundesregierung und der Deutsche Bundestag sowie die Aufsichtsbehörden, Bundesanstalt für Finanzdienstleistungsaufsicht und Deutsche Bundesbank. Die Bundesbank ist eine weisungsfreie Behörde (vgl. Art 88 GG; $\mathbb{S} 12$ BundesbankG), die nach dem Kreditwesengesetz $(\mathbb{S} 7 \mathrm{KWG}$ ) für die Bankenprüfung vor Ort zuständig ist und der BaFin Berichte der Prüfungen zuleitet. Die BaFin wiederum ist eine weisungsgebundene Behörde, die nach dem Finanzdienstleistungsaufsichtsgesetz zum einen der Rechts- und Fachaufsicht des Bundesministeriums der Finanzen unterliegt ( $\mathbb{S}$ 2 FinDAG) und zum anderen von einem Verwaltungsrat kontrolliert wird, der sich aus Vertretern von Bundesministerien, Bundestagsabgeordneten und Interessengruppenvertretern zusammensetzt ( $\mathbb{} 7$ FinDAG). Trotz der ministeriellen Fachaufsicht genießt die BaFin eine gewisse Unabhängigkeit, wenn man die Dimensionen der regulatorischen, aufsichtlichen, institutionellen und budgetären Selbstständigkeit betrachtet (vgl. Quintyn u. Taylor 2004, S. 4). Ein weites Vollzugsermessen besteht nicht nur in der Wahrnehmung der Aufsicht, sondern auch bei Verhandlungen auf europäischer Ebene, auf der die BaFin an den Implementierungsvorgaben für Regulierungsregime mitwirkt. Die Unabhängigkeit in der Aufsichts- und Regulierungsarbeit der BaFin wird durch institutionelle Faktoren, wie den Status als Anstalt des öffentlichen Rechts, und budgetäre Faktoren in Form der Umlagefinanzierung ergänzt (vgl. Handke 2010, S. 122). Die Autonomie der BaFin erklärt sich durch ihre Entstehung in einer Phase, in der es einen internationalen Trend zur Schaffung von „agencies“, wie der britischen Financial Services Authority (FSA) gab, nach deren Muster die deutsche Allfinanzaufsicht konstruiert wurde (vgl. Fach 2008). Dennoch ist die BaFin aufgrund der ministeriellen Fachaufsicht, die praktisch nahezu unumschränkt ist, nicht völlig unabhängig (vgl. Döhler 2002). Dies betrifft formal die Organisation und die interne Arbeitsweise der Behörde ebenso wie ihre Aufsichtsarbeit, sodass „the govern-

2 Das Leitbild des „mündigen Verbrauchers“ findet sich in verschiedenen Politikfeldern, so zum Beispiel im Lebensmittelbereich, der Gesundheits- oder auch in der Finanzmarktpolitik. 
ment [can] override or preempt, at no cost to itself, supervisory actions directed at troubled banks, thus keeping such banks open and risking higher costs to society in the future" (Quintyn u. Taylor 2004, S. 7). Die Arbeit der BaFin ist damit in hohem Maße von der Regierung und deren Verhalten abhängig.

Die Bundesregierung tritt im Politikfeld in erster Linie durch das Bundesministerium der Finanzen in Erscheinung, das unter anderem für Fragen der Haushaltspolitik, Währungspolitik, Finanzmarktregulierung und Finanzmarktaufsicht zuständig ist. Dem Ministerium obliegt die Aufgabe, den gesetzlichen Rahmen für den Finanzmarkt zu schaffen. Es ist daher zum Beispiel ermächtigt, Bestimmungen über die Eigenkapitalausstattung von Banken $(\mathbb{S} 10 \mathrm{KWG})$ zu regeln oder die Vergütungssysteme von Bankvorständen zu regulieren (\$25a KWG). Zahlreiche Aufgaben muss das BMF jedoch nicht selbst übernehmen, sondern kann der BaFin seine gesetzliche Ermächtigung übertragen, die diese dann - häufig im Benehmen mit der Bundesbank - im Rahmen der Gesetze ausfüllt.

Das BMF verfügt für seine Zuständigkeiten über sechs Referate, die in der Abteilung VII für Regulierungs- und Aufsichtsfragen von Banken, Versicherungen und Wertpapierwesen verantwortlich sind. Daneben existiert ein spezielles Referat für Grundsatzfragen der Finanzdienstleistungsaufsicht und die Rechts- und Fachaufsicht über die BaFin. Dieses Referat VII B 6 (Stand 2010) bzw. VII A 7 (Stand Juli 2011) ist überwiegend mit der Wahrnehmung der Rechts- und Fachaufsicht über die BaFin befasst, das heißt mit der Kontrolle der Behörde in den Bereichen Organisation, Personal und Haushalt (Interviewnachweis 2). Das Weisungsrecht in inhaltlichen Fragen wird durch die jeweiligen Fachreferate der Unterabteilung VII B ,zielorientiert [,...] vertrauensvoll [und mit dem Belassen der Erfüllung von] Aufgaben in eigener Zuständigkeit" der Behörde wahrgenommen (BMI 2008, S. 2). Dem BMF steht ein Bündel von Maßnahmen zur Verfügung, um das rechtmäßige und zweckmäßige Verwaltungshandeln der BaFin sicherzustellen, wobei Berichts- und Informationspflichten zu den wichtigsten Elementen zählen. Je stärker es aber nicht nur eine nachträgliche Kontrolle ausübt, sondern auch im Vorfeld steuernd eingreift, desto stärker spielt „die Arbeitsbelastung der aufsichtsführenden Referate eine Rolle“ (BMI 2008, S. 5). Es kommt damit zu einem Trade Off zwischen der intendierten Autonomie der Behörde und dem nach wie vor vorhandenen Interesse des Ministeriums, bei Bedarf kontrollierend und steuernd tätig werden zu können (vgl. Aulich 2010, S. 214). 
Abbildung 1: Anzahl der Mitarbeiter des BMF (1991 bis 2009)

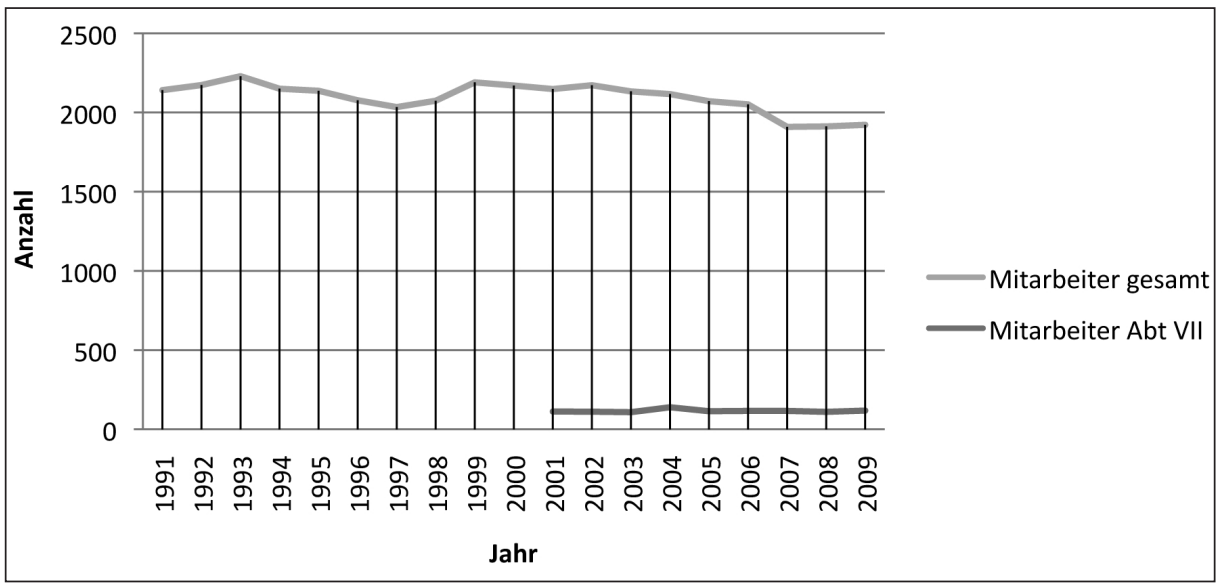

Quelle: Eigene Darstellung nach Deutscher Bundestag 2009a; BMF 20106.

Die personelle Ausstattung des BMF stellt in diesem Sinne einen limitierenden Faktor der Fachaufsicht dar. Angesichts begrenzter Budgets der öffentlichen Hand unterliegen Verwaltungen aller Körperschaften einem Einspardruck, der sich vor allem in Stellenkürzungen zeigt. Für die Bundesverwaltung gilt seit 1993 eine haushaltsgesetzliche Regelung, nach der in jedem Jahr 1,5 Prozent der Stellen zu reduzieren sind. ${ }^{3}$ Besondere Relevanz besitzt diese Reduktion in den Bundesministerien, die mit immer weniger Personal immer mehr Aufgaben in ihrem Politikbereich erledigen müssen. Das BMF bildet dabei keine Ausnahme, wie die Personalentwicklung der vergangenen Jahre (Abbildung 1) zeigt.

$\mathrm{Zu}$ den wesentlichen Aufgaben des BMF gehört im Politikfeld die Schaffung des Rechtsrahmens für Regulierung und Aufsicht. Dies umfasst die Erarbeitung von Gesetzen und die Entwicklung eher technischer Standards, wie der Berechnung und Bewertung der risikoadäquaten Eigenkapitalunterlegung von Banken und Versicherungen. Letzteres findet zunehmend auf europäischer oder internationaler Ebene statt, wie die Versicherungsregulierung durch Solvency II in der European Insurance and Occupational Pensions Authority (EIOPA) ${ }^{4}$ und die Bankenregulierung durch Basel III im Baseler Ausschuss für Bankenaufsicht zeigen. Seit 2002 werden derartige Aufgaben durch die BaFin wahrgenommen, die in den entsprechenden Gremien deutsche Interessen allein oder in Zusammenarbeit mit der Deutschen Bundesbank vertritt. Damit schafft die BaFin de facto selbst Regeln, die sie anschließend im nationalen Rahmen implementieren muss und erfüllt hierbei eine Aufgabe, die ursprünglich dem BMF oblag. Eine Programmentwick-

3 Von der Personaleinsparvorschrift wurde nur im Jahr 2010 abgewichen, in dem nur 1,0 Prozent der Stellen reduziert werden mussten. Von der ursprünglichen Planung, die Einsparverordnung auslaufen zu lassen, wurde wieder abgerückt, sodass auch in den kommenden Jahren der Personalbestand der Bundesverwaltung abnehmen wird (vgl. BMF 2010a).

4 Die European Insurance and Occupational Pensions Authority (EIOPA) ging zum 01.01.2011 aus dem Committee of European Insurance and Occupational Pensions Supervisors (CEIOPS) hervor. 
lungsfunktion als „Entscheidungsvorbereitung“ (Mayntz 1978, S. 184) nimmt das Ministerium demnach bereits seit einigen Jahren nicht mehr wahr, sondern beschränkt sich auf die selektive Kontrolle der Arbeit, die die BaFin als deutsche Repräsentantin leistet. Erst bei den aktuellen Verhandlungen zum neuen Bankenregime Basel III hat sich das BMF stärker in die Entstehungsprozesse eingebracht, da nach der Finanzkrise die Politisierung dieses Regelungsbereichs enorm zugenommen hat (vgl. Interviewnachweis 3 ).

Mit der Finanzkrise musste das BMF jedoch nicht nur die Programmvorbereitung, sondern auch die eigentliche Programmformulierung in nennenswertem Umfang auslagern. Während die Erarbeitung von Verordnungen oder Gesetzesanpassungen schon länger von der BaFin übernommen wird (vgl. Interviewnachweis 4), führte die Krise dazu, dass Gesetzentwürfe durch private Auftragnehmer verfasst wurden. Zentrale Programme wie das Finanzmarktstabilisierungsgesetz und dessen Fortentwicklungen verfasste die Rechtsanwaltskanzlei Freshfields Bruckhaus Deringer, Teile des Einlagensicherungsgesetzes erarbeitete die Kanzlei White\&Case und als Berater zu Gesetzentwürfen im Kreditwesenbereich wurde die Kanzlei Linklaters beauftragt (vgl. Deutscher Bundestag 2009). Das BMF hatte während der Finanzmarktkrise zwar weiterhin die Aufgabe, gesetzliche Regelungen auf den Weg zu bringen, die den Finanzmarkt stabilisieren. Einen Großteil der Gesetze konnte es jedoch nicht selbst vorbereiten, sondern musste sich der „Söldner des Rechts“ (Kurz 2009) bedienen, weil im Ministerium bereits seit einigen Jahren sowohl die personelle Ausstattung als auch die Fachkompetenz für die komplexe Materie fehlen (vgl. Lütz 2002; Döhler 2011).

Obgleich die Mitarbeiter des BMF nicht die nötigen Kenntnisse in den ungewohnten Rechtsbereichen hatten, wäre die Unterstellung eines grundsätzlichen Mangels an Fachkompetenz unzutreffend. Das Fachpersonal der Abteilung VII gilt bei Finanzunternehmen, Branchenverbänden und Politikern gleichermaßen als „durchaus sehr kompetent für den Normalbetrieb“ (Interviewnachweis 5). Ein größeres Problem besteht hingegen in der quantitativen Personalausstattung der 2001 geschaffenen Abteilung, die - obwohl sie mit ca. 118 Mitarbeitern in 19 Referaten eine der größeren des Ministeriums ist - an der Kapazitätsgrenze arbeitet (BMF 2010b). Mit Aufgaben der Finanzmarktregulierung und -aufsicht sind mithin nur 37 Mitarbeiter betraut, von denen sieben ausschließlich für die Bereiche Organisation, Personal und Haushalt der BaFin zuständig sind. Im Gegensatz zum übrigen Ministerium wurden in der Abteilung zwar keine Stellen abgebaut, allerdings hat sich der Aufgabenumfang während der Finanzkrise erhöht, sodass die Abteilung VII mit ihrem Mitarbeiterbestand bereits vor der Krise „total auf den Punkt gebracht [und] total ausgeblutet“ (Interviewnachweis 6) war und ministerielle Aufgaben nicht mehr selbst oder nicht in angemessener Zeit erfüllen konnte. Diese Einschätzung, zu der von Seiten des BMF keine Stellungnahme abgegeben wurde, teilten noch 2010 ausnahmslos alle Interviewpartner.

Vor diesem Hintergrund muss davon ausgegangen werden, dass das BMF den Bereich der Finanzmarktpolitik formal zwar gestaltet, faktisch aber in hohem Maße auf die Unterstützung von nachgeordneten Behörden und externen Vertragspartnern angewiesen ist. Letzteres ist - abgesehen von möglichen Legitimationsproblemen, die hier nicht weiter betrachtet werden sollen - allenfalls in Aus- 
nahmefällen unproblematisch, um Spitzen in der Auslastung zu kompensieren, nicht jedoch wenn sich die Abhängigkeit des Ministeriums aufgrund struktureller Merkmale zu verstetigen droht. Hier zeigt sich eine Diskrepanz zwischen dem selbstgesteckten Anspruch des BMF und seinen Möglichkeiten definierte Ziele zu erreichen. Der Ausweg über die Beauftragung privater Rechtsanwaltskanzleien zeigt darüber hinaus, dass das BMF auch der BaFin diese Aufgabe nicht zutraut oder nicht übertragen will. Im Folgenden wird die Delegationsbeziehung zwischen BMF und BaFin mit ihren spezifischen Delegationsproblemen im Mittelpunkt stehen, um die Frage zu klären, ob es neben den Schwächen in der Politikformulierung auch Probleme bei der Steuerung der BaFin gibt.

\section{Ministerielle Steuerungsinteressen und Delegationsprobleme}

Das BMF wird als federführendes Ministerium in der Finanzmarktpolitik von nachgeordneten Behörden unterstützt. Von diesen ist die BaFin die größte und wichtigste, da sie für den gesamten Bereich der Finanzmarktaufsicht - mit Ausnahme der Vor-Ort-Prüfung von Banken - zuständig ist und zudem die deutsche Verhandlungsführung in verschiedenen internationalen und europäischen Gremien übernimmt. Aufgrund seiner Letztverantwortlichkeit im Politikfeld und wegen der gewerbepolizeilichen Eingriffsrechte, über die die BaFin verfügt, übt das BMF neben der Rechtsaufsicht auch die Fachaufsicht über die BaFin aus ( $\$ 2$ FinDAG). Das Ministerium ist damit für die Handlungen der Behörde zumindest politisch verantwortlich. Eine rechtliche Verantwortlichkeit besteht hingegen nicht vollumfänglich, da die BaFin als Anstalt des öffentlichen Rechts eine eigene Rechtspersönlichkeit besitzt. Seit der Gründung der BaFin im Jahr 2002 hat eine Entwicklung im Verhältnis zwischen Ministerium und nachgeordneter Behörde stattgefunden, die sich mit Modellen der Delegationstheorie beschreiben lässt. Die BaFin ist in dieser Beziehung der Auftragnehmer, das Finanzministerium der Auftraggeber. Beide sind arbeitsteilig für den Bereich der Finanzmarktpolitik zuständig und nehmen entsprechende Aufgaben wahr.

Im Zusammenhang mit der Errichtung von unabhängigen oder teilweise unabhängigen Regulierungsbehörden (agencification) traten in der Vergangenheit nicht nur Fragen nach Effizienz und demokratischer Legitimation auf, sondern auch nach der Steuerbarkeit solcher Agenturen innerhalb von Delegationsbeziehungen (vgl. Christensen et al. 2008; Bach et al. 2010). Für die Betrachtung dieses Verhältnisses eignen sich vor allem zwei aus der Ökonomie entlehnte Konzepte, zum einen die Prinzipal-Agenten-Theorie, zum anderen die Trust-Theorie (vgl. u. a. Gilardi u. Braun 2002; Alter 2008; Handke 2010). Die Anwendung beider Ansätze soll mit einigen knappen Ausführungen die Konfliktlinie in der Beziehung zwischen BMF und BaFin skizzieren und mit den Herausforderungen der ministeriellen Fachaufsicht verknüpfen.

Eine simple Prinzipal-Agent-Beziehung zeichnet sich dadurch aus, dass in einer arbeitsteiligen Konstellation zwischen zwei Akteuren die Erfüllung einer Aufgabe per Vertrag von einem Auftraggeber (Prinzipal) an einen Auftragnehmer (Agent) übertragen wird. Der Vertrag beinhaltet die Pflichten beider Partner und schreibt dem Agenten die Auftragserfüllung in Interessenvertretung des Prinzipals vor. 
Eine solche Vertragsbeziehung, wie sie auch zwischen BMF und BaFin besteht, ist in der Regel durch Informationsasymmetrien gekennzeichnet. Der Agent ist dadurch in der Lage, seinen Informationsvorsprung gegenüber dem Prinzipal zu seinen Gunsten, das heißt zur Maximierung seines eigenen Vorteils, auszunutzen. Beide Akteure verfolgen in der Modellvorstellung individuelle, nutzenmaximierende Strategien, die dazu führen können, dass Leistungen - zu Lasten des Vertragspartners - mit minimalem Aufwand erbracht werden. Der Anreiz des Agenten besteht darin, seinen Auftraggeber zu betrügen (moral hazard) oder ihn zumindest mit den geringstmöglichen Kosten für sich selbst zufriedenzustellen (Gilardi u. Braun 2002, S. 147-148). Dem Prinzipal steht zwar die Möglichkeit der Kontrolle des Auftragnehmers offen, allerdings stellt Überwachung einen Kostenfaktor dar, der nicht nur den Nutzen des Auftraggebers mindert, sondern im Falle einer vollständigen Kontrolle auch den Sinn von Arbeitsteilung infrage stellt. Abweichende Zielvorstellungen zwischen den Vertragspartnern können letztlich nicht gänzlich verhindert werden und die Gefahr des Kontrollverlustes über den Agenten, also eines „agency loss“ bzw. „bureaucratic drift" in der Verwaltung, bleibt bestehen (McCubbins et al. 1987).

Um ein abweichendes Verhalten des Agenten dennoch zu verhindern oder zu minimieren, stehen dem Prinzipal eine Reihe von ex ante wirkenden Maßnahmen zur Verfügung. Diese sind neben vertraglichen Ausgestaltungen des Auftrages vor allem sorgfältige Auswahlprozesse für den Agenten, die Festlegung von Berichtspflichten und die Definition von Kontrollbefugnissen durch den Prinzipal (vgl. Kiewiet u. McCubbins 1991, S. 27). Sollten alle Maßnahmen scheitern, bleiben dem Prinzipal als letzte Mittel noch immer die Abänderung oder Auflösung des Vertrags bzw. die Einsetzung eines alternativen Agenten (vgl. Handke 2010). Im Fall der BaFin steht dieser Weg dem BMF jedoch nicht ohne Weiteres offen, da die Delegationsbeziehung zwischen beiden Akteuren als Trust-Beziehung einer speziellen Form des Prinzipal-Agent-Musters mit weiteren Randbedingungen entspricht. Drei wesentliche Unterschiede zwischen den Delegationsmodi liegen, wie Tabelle 1 zeigt, in der Einbeziehung Dritter, der Reichweite des Auftrages und der Anwendung von Sanktionsmaßnahmen. Ein Treuhänder berücksichtigt in seiner Arbeit nicht nur die Interessen des Prinzipals, sondern auch die eines begünstigten Dritten, für den er in einem breiten Kompetenzraum und nicht nur im Einzelfall zu handeln ermächtigt ist. Durch den Einfluss des dritten Akteurs stehen dem Prinzipal zudem nicht mehr die Sanktionsmaßnahmen zur Verfügung, die er ohne Rücksicht auf andere verwenden könnte. 
Tabelle 1: Merkmale von Auftragnehmern in Delegationsbeziehungen

\begin{tabular}{|l|l|l|}
\hline & Agent & Treuhänder \\
\hline Auftrag & Leistung zugunsten des Prinzipals & Leistung für Begünstigten \\
\hline $\begin{array}{l}\text { Auswahlkriterien durch } \\
\text { Prinzipal }\end{array}$ & $\begin{array}{l}\text { Präferenzkongruenz; erwartete } \\
\text { Nutzenmaximierung für Prinzipal }\end{array}$ & $\begin{array}{l}\text { Expertise und Reputation; } \\
\text { erwartete Nutzenmaximierung } \\
\text { für Begünstigten }\end{array}$ \\
\hline Verhaltensanleitung & Anweisung des Prinzipals & Professionelle Standards \\
\hline Reichweite des Auftrags & $\begin{array}{l}\text { Ermächtigung für eine Einzel- } \\
\text { aufgabe }\end{array}$ & $\begin{array}{l}\text { Ermächtigung für einen weiten } \\
\text { Aufgabenkomplex }\end{array}$ \\
\hline Steuerungsmittel & Anweisung des Prinzipals & $\begin{array}{l}\text { Wünsche von Prinzipal und } \\
\text { Begünstigtem }\end{array}$ \\
\hline Agenturproblem & $\begin{array}{l}\text { Minimalleistung bzw. verdeckte } \\
\text { Nichtleistung; „agency loss“ }\end{array}$ & $\begin{array}{l}\text { Minimalleistung bzw. verdeckte } \\
\text { Nichtleistung; „,agency loss“ }\end{array}$ \\
\hline Ausweg des Prinzipals & Vertragsänderung; Entlassung & Umgehung; Entlassung \\
\hline
\end{tabular}

Quelle: Eigene, veränderte Darstellung nach Handke 2010, S. 119-120.

Der Treuhänderstatus der BaFin, die mit Ermächtigung des BMF im Interesse von Marktakteuren und Verbrauchern tätig ist, um Vertrauen und Systemstabilität sicherzustellen, verhindert also einen unbeschränkten Zugriff durch das Ministerium. Damit steht diesem die Auswahl eines alternativen Agenten oder die Entbindung der BaFin von ihren Aufgaben nur dann offen, wenn der BaFin größeres Versagen und der Verstoß gegen die Interessen von Prinzipal und begünstigten Dritten nachgewiesen werden kann (vgl. Handke 2010). Fehlerhaftes Verhalten versucht die BaFin jedoch schon aus Eigennutz zu vermeiden, da sie ansonsten nicht nur ihrer Reputation als Fachbehörde schaden, sondern auch die Realisierung grundlegender Organisationsziele gefährden würde. $\mathrm{Zu}$ diesen Zielen, die die BaFin als bürokratische Organisation ebenso verfolgt wie das BMF oder die Bundesbank, gehören in erster Linie der Wunsch nach Fortbestand und Ausbau der Behörde sowie die Maximierung des Budgets (vgl. Niskanen 1979). Aus diesem Interesse resultiert die Strategie von Behörden, ihren Aufgabenbereich als unverzichtbar und ressourcenintensiv - eventuell auch stärker als für die Aufgabenerfüllung nötig - darzustellen, um dadurch mehr finanzielle und personelle Mittel zu erhalten. Diese Mittel werden in einer pessimistischen Interpretation des Behördenhandelns für individuelle Annehmlichkeiten der Mitarbeiter verwendet. In der Regel werden Ressourcen der Organisation jedoch zur Erreichung von PolicyZielen und zur Festigung der Behördenposition im Politikfeld verwendet (vgl. Downs 1967). Zu dieser Verankerung im Politikfeld gehört auch, dass sich eine Behörde und ihre Mitarbeiter möglichst aus öffentlich ausgetragenen Konflikten heraushalten, um nicht Gegenstand politischer oder medialer Kritik zu werden. Dieses Verhalten ist an das „bureau shaping“ angelehnt, das auf eine möglichst komfortable Aufgabenerfüllung innerhalb der Behördenarbeit abzielt (vgl. Dunleavy 1991).

Verfolgen BMF und BaFin jeweils diese drei Strategien, ist ein Konflikt zwischen beiden Behörden möglich, sobald Zielvorstellungen hinsichtlich der Regu- 
lierung und Aufsicht abweichen. Aus diesem Grund wurden bereits bei der Errichtung der BaFin ex ante wirkende Bestimmungen zur Steuerung der Behörde in das FinDAG (u. a. $\mathbb{S} 2,3,7,8$ ) aufgenommen, die einen Hinweis auf die Steuerungsinteressen des BMF geben. Mit den „Grundsätzen für die Ausübung der Rechtsund Fachaufsicht des Bundesministeriums der Finanzen (BMF) über die BaFin“ wurden diese noch einmal ergänzt und präzisiert (BaFin 2010). Unter Steuerung wird dabei zum einen die Möglichkeit verstanden, durch Anweisungen Ziele vorzugeben, die regulatorisch erreicht werden sollen, zum anderen Wege zu bestimmen, mit denen die Ziele durch aufsichtliches Handeln erreicht werden (vgl. Eichhorn et al. 1985, S. 345-346).

$\mathrm{Zu}$ den Steuerungsinteressen, die das BMF verfolgt, zählen verschiedene Überlegungen in Bezug auf die Organisation und die Tätigkeit der BaFin bei Aufsicht und Regulierung. Durch ihre Konstruktion als Anstalt des öffentlichen Rechts ( $\mathbb{S}$ 1 FinDAG) und die Finanzierung durch ein Gebührensystem ( $\mathbb{S} 14$ FinDAG), ist die BaFin dem Behördentypus der unabhängigen Agentur sehr nah (vgl. Aulich 2010). Die Zugriffsmöglichkeiten des Ministeriums sind somit begrenzt und finden im Rahmen einer politisch intendierten Selbstständigkeit der BaFin statt. Gründe für die Einrichtung einer relativ unabhängigen Behörde waren einerseits, auf diese Weise den Anschein reiner, objektiver Fachlichkeit für die Wahrnehmung der Aufsicht zu erwecken, andererseits aber auch eine Institution im Politikfeld zu etablieren, die unbequeme Entscheidungen treffen kann, ohne dass Kritik daran direkt auf das Ministerium zurückfällt (vgl. Thatcher 2002). Dieses „blame shifting" ist als wesentlicher Grund für die Errichtung unabhängiger oder quasi-unabhängiger Agenturen zu erachten und besitzt aus der Nutzenüberlegung des BMF auch für die BaFin Gültigkeit (vgl. Ebinger u. Schmitt 2010). Gleichzeitig ist das BMF daran interessiert, dass die BaFin ihre Aufgaben so erfüllt, dass möglichst wenige Konflikte und Störungen auftreten und dadurch zwingende Problemlösungsaufgaben für das Ministerium entstehen. Aus Sicht eines Ministeriums mit begrenzten Ressourcen und Informationsverarbeitungskapazitäten ist die Arbeit einer nachgeordneten Behörde ideal, wenn sie wenige eigene Ressourcen bindet und sich die Fachaufsicht ohne größeren Zeitaufwand ausüben lässt (vgl. BRH 2005, S. 17). Dies bedeutet, dass sich die Wahrnehmung der Fachaufsicht auf allgemeine Kontrolle und fallweise Steuerung beschränken kann und direkte Eingriffe per Weisung nur in Ausnahmefällen vorkommen (vgl. Döhler 2007a, S. 238-247). Im Fall der BaFin zeigte sich jedoch, dass das BMF seine Steuerungsfähigkeit, also die Möglichkeit, regulatorische Ziele zu definieren und aufsichtliche Maßnahmen zur Zielerreichung durch Anweisung zu bestimmen, in Gefahr sah. Ausgelöst durch das vermeintlich zu eigenständige Agieren der BaFin während der Basel II-Verhandlungen, die Einbindung in europäische Gremien und ein sehr selbstbewusstes Auftreten der Behördenspitze, ergriff das BMF Maßnahmen, um die BaFin in ihrer Emanzipationsentwicklung zu beschränken (vgl. u. a. Sturm et al. 2002; Döhler 2007). Zu diesen Schritten gehören in erster Linie der Umbau der Leitungsstruktur von einer Präsidial- zu einer Kollegialstruktur und die detaillierte Fixierung von Richtlinien zur Wahrnehmung der ministeriellen Rechts- und Fachaufsicht über die Behörde, die in der Logik der Prinzipal-Agenten-Theorie eine Vertragsänderung darstellen, jedoch gegenüber einem Treuhänder nur be- 
dingt wirksam sind (vgl. Handke 2010, S. 130). Das BMF, das „die Umsetzung der Richtlinien [...] nicht im Einzelfall überprüft" (Deutscher Bundestag 2006, S. 4), ist mit den Konkretisierungen der Fachaufsicht seinem Eigeninteresse nach einem möglichst geringen Ressourceneinsatz für den nachgeordneten Bereich gefolgt, denn es besteht für die BaFin eine Bringschuld gegenüber dem Ministerium, die jeden Bereich der aufsichtlichen und regelschaffenden Arbeit umfasst. Dieser Verpflichtung, die das BMF als vorgesetzte Behörde entlastet, ist die BaFin im Vorfeld und während der Finanzkrise nachgekommen. Am Beispiel der Bank Hypo Real Estate zeigte sich jedoch, dass die geringe Intensität der Fachaufsicht, die im Regelfall „nur das Abheften und Ablegen von Meldungen der BaFin durch Mitarbeiter des mittleren Dienstes“ (Interviewnachweis 7) umfasste, unzureichend war. Mit der Abteilung VII und vor allem dem dort angesiedelten Aufsichtsreferat VII B 6 bzw. VII A 7 ist das Ministerium zwar bemüht, die nachgeordnete Aufsichtsbehörde hinsichtlich der Regulierung an die Präferenzen des Hauses zu binden und zu steuern. Allerdings kommen Hood et al. zu der Einschätzung, dass „like private and other interests, bureaucratic and regulatory interests [are] often multiple and conflicting, not a monolithic bloc" (2003, S. 126). Dadurch zeichnet sich ein doppelter Konflikt zwischen BMF und BaFin ab, der die Steuerung in Bezug auf regulatorische Zieldefinitionen ebenso umfasst wie das aufsichtliche Tagesgeschäft. Diese Schlussfolgerung lässt zumindest der Bericht eines ehemaligen BaFin-Mitarbeiters der Leitungsebene zu, der die Erteilung von ministeriellen Einzelfallweisungen bei der Prüfung bestimmter Institute bestätigt (vgl. Interviewnachweis 8). Derartige Eingriffe, mit denen bereits geplante Prüfungen untersagt wurden, sind zwar im Rahmen der Fachaufsicht durchaus möglich, laufen aber dem Interesse der BaFin nach aufsichtlicher Selbstständigkeit als spezifischem Organisationsziel zuwider.

Wie bei jeder bürokratischen Organisation kann auch bei der BaFin davon ausgegangen werden, dass „bureaucrats [...] constitute an interest group seeking to shape public policy and organization, though what they seek to maximize is much debated“ (Hood et al. 2003, S. 125-126). Aufgrund dieser Logik ist es für die BaFin eine rationale Strategie, Aufsicht und Regulierung als zentrale und unverzichtbare Elemente der Finanzmarktpolitik darzustellen (vgl. Sanio 2002).

Aufseiten der BaFin besteht damit das Interesse an einer strengen Aufsicht und der allgemeinen Akzeptanz eines erhöhten Aufsichtsbedarfs - der sich zum Beispiel durch die Einführung interner Risikomodelle für Banken und Versicherungen ergibt, die Institute anstelle der Standardmodelle in Basel II oder Solvency II verwenden dürfen -, da dies die Existenz und den Ausbau der Behörde rechtfertigen kann. Es ist das Ziel von Aufsicht, Regulierung so zu implementieren und zu überwachen, dass daraus ein möglichst stabiles Finanzsystem resultiert, in dem das Ausbleiben von Bankenzusammenbrüchen als Indiz für das Funktionieren der Aufsicht interpretiert wird. Das Bundesaufsichtsamt für das Kreditwesen (BaKred) hatte es mit dieser Strategie zwischen 1988 und 2001 geschafft, die Anzahl seiner Planstellen um 270 Prozent zu steigern und auch die BaFin konnte den Mitarbeiterbestand von 2002 bis 2005 um 30 Prozent erhöhen (vgl. Kaserer 2006, S. 69, 73). Die BaFin, die auch für das Jahr 2011 etwa 240 zusätzliche Stellen bekommen hat, ist damit eine wachsende Behörde, die ihren Mitarbeiterbe- 
stand um 13 Prozent auf etwa 2000 Mitarbeiter ausbauen konnte (vgl. BaFin 2010a; Handelsblatt 01.03.2011). Für die ministerielle Fachaufsicht bedeutet dies eine weitere Verschlechterung der Relation zwischen beaufsichtigenden und beaufsichtigten Mitarbeitern.

Auch dies begünstigt das Bestreben der BaFin, sich der Kontrolle des BMF zumindest so weit zu entziehen, dass die Anmeldung eines hohen Aufsichtsbedarfs durch das Ministerium nicht infrage gestellt wird. Dieses Ziel kann durch eine starke fachliche Kompetenzbündelung der BaFin erreicht werden, die ihr einen Informationsvorsprung gegenüber dem BMF gibt. Zum Ausdruck kommt dieser Vorsprung aber auch bei der Erarbeitung von Regulierungsregimen, die wie Basel II und Solvency II einen hohen technischen Komplexitätsgrad erreichen, sodass das BMF sich weitgehend auf die Expertise der BaFin verlassen muss. Die Folge ist ein Konflikt zwischen BMF und BaFin, der entsteht, weil sich die Aufsichtsbehörde von der faktischen Fachaufsicht entfernen kann, das BMF aber die Möglichkeit erhalten will, die Fachaufsicht bei Bedarf mit beliebiger Intensität ausüben zu können. In der Finanzkrise zeigte sich aber genau hier die Schwäche des BMF, das die Aufsicht über die öffentlich unter Druck geratene BaFin nicht intensivieren konnte (vgl. Döhler 2011). Dies stellt ein klassisches Prinzipal-AgentenProblem dar, das durch die Beteiligung weiterer Akteure - insbesondere der Marktteilnehmer, aber auch der Bundesbank - zu dem beschriebenen, komplexeren Delegationsproblem einer Trust-Beziehung wird. Aus diesem Grund ist davon auszugehen, dass das BMF einen Kontroll- und Steuerungsverlust über die BaFin, der aus der ursprünglich politisch intendierten Teilautonomie der Behörde, ihrer sich damit eröffneten Fähigkeit zur Emanzipation und ihrem Expertisevorsprung gegenüber dem Ministerium resultiert, selbst wahrnimmt (vgl. Frach 2008; Handke 2010; Döhler 2011).

\section{Verantwortlichkeit des Ministeriums und Verantwortung der BaFin}

Die Beziehung zwischen BMF und BaFin birgt nicht nur ein Prinzipal-Treuhänder-Problem, sondern zeichnet sich gleichzeitig durch ein massives Auseinanderfallen von Verantwortung und Verantwortlichkeit aus. In Anlehnung an die Dichotomie von Niklas Luhmann beschreibt die Übernahme von Verantwortung eine Individual- oder Organisationsleistung, bei der Informationen verarbeitet, verdichtet und unter „Absorption von Unsicherheit“ an andere Individuen oder Organisationen weitergegeben werden (Luhmann 1994, S. 178). Dagegen betrifft Verantwortlichkeit kein Element des direkten Kommunikationsprozesses, sondern eine Folge fehlerhafter Informationsweitergabe. Vor allem in formalen Organisationen findet sich Verantwortlichkeit als sanktionsbewehrte „Rechenschaftspflicht für Fehler", die als vertrauensschaffendes Moment der Kommunikation akzeptiert wird (Luhmann 1994, S. 179). Während Verantwortung in Organisationen wie Ministerien oder anderen Behörden vor allem auf der Arbeitsebene übernommen wird, befinden sich die Träger der Verantwortlichkeit an der Spitze und müssen nach außen Rechenschaft ablegen (vgl. Luhmann 1994, S. 183).

Im Fall der Aufgabenteilung zwischen BMF und BaFin findet das beschriebene Muster zwar nicht innerhalb einer Organisation statt, sondern zwischen zwei Be- 
hörden. Allerdings bestehen Parallelen zu der Differenzierung von Verantwortung und Verantwortlichkeit innerhalb einer einzelnen Organisation. Die BaFin ist für die Beaufsichtigung und Prüfung von Finanzunternehmen zuständig und verrichtet damit eine Arbeit, die im besten Sinne Verantwortung bedeutet, da Informationen aus einer Vielzahl von Quellen gesammelt und verarbeitet werden. Aus diesen Informationen werden wiederum Schlüsse gezogen, die Aussagen über die Solvenz einer Bank oder die Stabilität des gesamten Finanzsystems zulassen. Entsprechende Einschätzungen werden im Rahmen der Berichtspflicht der BaFin regelmäßig an das BMF weitergeleitet (vgl. BaFin 2010). Das Ministerium verwendet diese Informationen als Grundlage für rechtliche Maßnahmen, die im Bereich der Finanzmarktpolitik getroffen werden, und ist damit letztlich verantwortlich für alle Entscheidungen. Diese Verantwortlichkeit trifft nicht nur das BMF als gesamte Organisation, sondern über den Weg der internen Hierarchie in besonderer Weise die Leitung des Hauses. Um der BaFin nicht „ausgeliefert“ zu sein, verfügt das BMF über die Fachaufsicht, die ihm das Recht gibt, Weisungen zu erteilen und Informationen jeder Art abzurufen (Frach 2008, S. 116-117). Mit Quartals- und Sonderberichten kommt die BaFin der ihr zugewiesenen Informationspflicht nach, ohne dass die Abteilung VII oder die Leitung des BMF daraus bereits die Notwendigkeit für eigene Aktivitäten ableiten (vgl. Deutscher Bundestag 2009, S. 110-111). Die Rolle einer „Ober- oder Superaufsichtsbehörde“ für den Finanzsektor nimmt das Ministerium nicht ein, da der Umfang aufsichtlicher Daten für eine Nachprüfung jedes Einzelfalls zu groß wäre und regelmäßige Doppelprüfungen dem Sinn von Arbeitsteilung widersprechen würden (Deutscher Bundestag 2009, S. 102). Vielmehr zeigt sich in der täglichen Zusammenarbeit von BaFin und BMF, dass die „fiktive Unterstellung [...], daß eine Information zutrifft, wenn sie von der zuständigen Stelle kommt" (Luhmann 1994, S. 179), Einzug in das reale Behördenhandeln gehalten hat. Das BMF nimmt alle Berichte - auch Berichte über Sonderprüfungen - von BaFin und Deutscher Bundesbank lediglich zur Kenntnis und vertraut ohne weitere Prüfung auf deren Richtigkeit. Auf Mitteilungen der BaFin wird damit nur dann in besonderer Weise reagiert, wenn diese das Ministerium explizit auf einen außergewöhnlichen Handlungsbedarf hinweist oder Unterstützung erbittet (vgl. Deutscher Bundestag 2009, S. 112). Das BMF als Prinzipal bringt der BaFin als Auftragnehmer in diesem Sinne einerseits ein hohes Maß an Vertrauen entgegen und verlässt sich weitgehend auf die korrekte Informationsverarbeitung und Verantwortungsübernahme der Behörde, andererseits begibt es sich aber auch in die Gefahr, für unerwartete Fehler der BaFin einstehen zu müssen.

Für die Performanz von BMF und BaFin ist das Auseinanderfallen von Verantwortung und Verantwortlichkeit gravierend. Während ein Ministerium, in dessen Politikbereich eine vollständig unabhängige Regulierungsbehörde ihre Arbeit verrichtet, in Krisenfällen eigenes Versagen von sich weisen und die Schuld für Fehler auch öffentlich der Behörde anlasten kann (blame shifting), ist diese Strategie für das BMF unwahrscheinlich. Die Weisungsbefugnis gegenüber der BaFin führt dazu, dass das Ministerium in der politischen Verantwortung steht und sich, vertreten durch den Minister, zum Beispiel gegenüber dem Parlament rechtfertigen muss. Eine solche Konstellation, in der das vorgesetzte Ministerium, indem es 
seine untergebene Behörde „zur Rechenschaft zieht, [...] zugleich seine eigene Verantwortlichkeit [aktualisiert], tendiert [...] nicht gerade zur Entdeckung von Fehlern, sondern eher zur Kollusion bei ihrer Verbergung “ (Luhmann 1994, S. 183). Das Beispiel HRE zeigt, dass ein entsprechendes Verhalten tatsächlich stattfindet und der BaFin durch das BMF keine Versäumnisse vorgeworfen werden. Die Leistungsfähigkeit der Finanzmarktpolitik als Gesamtaufgabe wird dadurch jedoch nicht befördert, sodass hierin ein Nachteil gegenüber einem System zu sehen wäre, in dem die Finanzaufsicht von einer weisungsfreien Behörde wahrgenommen wird (vgl. Burghof 2009). Als Treuhänder verfolgt die BaFin jedoch das Ziel, die eigene Reputation nicht zu schädigen, sodass sie gegenüber dem BMF kein reziprokes Verhalten zeigt und - um die Korrektheit des eigenen Handelns zu unterstreichen - auf Fehler des Ministeriums hinweist, soweit dies im Rahmen der Grundsätze zur Fachaufsicht möglich ist (vgl. Deutscher Bundestag 2009). Damit reagiert die Behörde mit einem „umgekehrten blame shifting " 5 und handelt damit gegen das Interesse des Prinzipals. Auch vor diesem Hintergrund lassen die speziellen Merkmale der Delegationsbeziehung zwischen BMF und BaFin, in der das Auseinanderfallen von Verantwortung und Verantwortlichkeit ein verschärfendes Moment darstellt, Konfliktpotenziale erkennen, die eine erhöhte ministerielle Achtsamkeit und Skepsis erklären.

Dem Ministerium ist die Abhängigkeit von der loyalen Zuarbeit seiner nachgeordneten Behörde allerdings bewusst, weshalb von der grundsätzlich unbeschränkten Fachaufsicht (vgl. Döhler 2002) nicht in einer Weise Gebrauch gemacht werden kann, die zu einer - längerfristigen - Konfrontation mit der BaFin führt. Ein moderater Einsatz der Weisungskompetenz ist wahrscheinlich, da dies der Kapazität des BMF entspricht und der BaFin den Eindruck von weitreichender Selbstständigkeit in Fachfragen vermittelt. Daraus ist zu erklären, dass es nur selten zu ausdrücklichen Weisungen an die BaFin kommt und das Ministerium vielmehr Wünsche äußert, die von der Aufsichtsbehörde möglichst erfüllt werden (vgl. Interviewnachweis 9). Hierbei zeigt sich seit dem Umbau der Leitungsstruktur zuweilen ein vorauseilender Gehorsam der BaFin gegenüber dem Ministerium, der einen expliziten Einsatz von Weisungen überflüssig macht und die Fachaufsicht eher als „Rute im Fenster“ (Mayntz u. Scharpf 1995, S. 29) erscheinen lässt. Allerdings hatten die beschriebenen Delegationsprobleme in den Jahren zuvor wiederholt zu Bemühungen geführt, die Handlungsfreiheit der BaFin einzuschränken und damit politisch intendierte Ermessensspielräume nachträglich zu begrenzen. Der Umbau der Behördenleitung, die allgemeine Intensivierung der Fachaufsicht und die Festlegung von speziellen Grundsätzen zur Ausübung der ministeriellen Aufsicht stellen einerseits grundsätzliche Korrekturversuche des Verhältnisses zwischen BMF und BaFin dar, andererseits waren die Maßnahmen auch gezielt auf Jochen Sanio als Präsident der BaFin gerichtet, der das Delegationsproblem zwischen beiden Behörden personifiziert (vgl. Handelsblatt 26.10.2009; Döhler 2011).

5 Für diese Formulierung bedanke ich mich bei einem anonymen Gutachter. 


\section{Kontrollverluste des BMF}

Wie gezeigt werden konnte, ist die Delegationsbeziehung zwischen BMF und BaFin anfällig für Konflikte und Steuerungsprobleme, allerdings lassen sich diese empirisch schwer nachweisen, da Maßnahmen der Fachaufsicht in der Regel nicht dokumentiert werden.

Die wissenschaftliche Literatur geht davon aus, dass der Richtlinienkatalog zur Fachaufsicht vor allem als Strafmaßnahme wegen der allzu selbstständigen Arbeit der BaFin verfasst wurde (vgl. u. a. Sturm et al. 2002; Döhler 2007; Quaglia 2008; Handke 2010b). Aktuellere Recherchen liefern jedoch zunächst keinen belastbaren Beleg dafür, dass die BaFin sich in den Gremien des europäischen Lamfalussy-Verfahrens oder in den Verhandlungen zum Bankenregime Basel II von der Steuerung durch das BMF irreversibel verselbstständigt hat. Vielmehr bestand an derartigen Verhandlungen bis zur Finanzkrise nur ein geringes Interesse, weshalb der BaFin und der Deutschen Bundesbank unter anderem die Arbeit im Baseler Ausschuss weitgehend ohne ministerielle Einflussnahme überlassen wurde (vgl. Deutsche Bundesbank 2010). Die Finanzkrise hat die Regelschaffung im Finanzmarkt jedoch zunehmend politisiert, sodass sich das BMF stärker damit befassen musste (vgl. Interviewnachweis 10). Dabei wurden jedoch die Grenzen der ministeriellen Steuerungskompetenz deutlich, die darin bestehen, dass das BMF die Arbeit der BaFin weder qualitativ noch quantitativ ausreichend kontrollieren kann (vgl. Döhler 2011). Das BMF ist aus diesem Grunde - wie auch im Fall von Basel II - erst mit einer zeitlichen Verzögerung in der Lage zu beurteilen, ob die BaFin im Sinne des Ministeriums gehandelt hat. Daher werden Steuerungs- und Kontrollverluste häufig nur ex post festgestellt und Maßnahmen erst mit einem gewissen Abstand zu den wahrgenommenen Delegationsproblemen ergriffen. Zwischen BMF und BaFin herrscht somit eine Informationsasymmetrie, die trotz der Ausdehnung von Berichtspflichten und Monitoring-Mechanismen zu einem faktischen Kontrollverlust des Ministeriums führt.

Im Rahmen des Delegationsverhältnisses zwischen den beiden Behörden hat zudem die Personalkonstellation in den Leitungsebenen von BaFin, BMF und Deutscher Bundesbank zu Spannungen geführt, die von einer institutionenbezogenen Betrachtung nicht erfasst werden. Das Verhältnis zwischen BaFin-Präsident Jochen Sanio und Finanzstaatssekretär Jörg Asmussen galt in den zurückliegenden Jahren als derart belastet, dass sogar von einem „Nichtverhältnis“ die Rede war, in dem der Kontakt zwischen beiden auf das Nötigste beschränkt wurde (Interviewnachweis 11). Verschiedene Quellen, vor allem Berichte von Akteuren aus dem Umfeld von Jochen Sanio, vermitteln das Bild eines Behördenleiters, der fachlich höchst anerkannt, persönlich jedoch weitgehend isoliert ist. Dabei war nicht nur die Zusammenarbeit mit Jörg Asmussen problematisch, sondern auch die mit dem ehemaligen Bundesbankpräsidenten Axel Weber und dem damaligen Finanzminister Peer Steinbrück. Im Zuge der HRE-Krise führte die Isolation Sanios dazu, dass der Präsident der BaFin eine Art Sündenbockfunktion übernehmen musste, während alle übrigen Akteure versuchten sich schadfrei zu halten. Vor allem Weber und Asmussen, sowie der ehemalige Abteilungsleiter für Wirtschafts- und Finanzpolitik im Bundeskanzleramt und derzeitige Bundesbank- 
präsident, Jens Weidmann, bildeten ein „eisernes Dreieck“, das für die jeweilig repräsentierte Behörde jeden Fehler bestritt (vgl. Deutscher Bundestag 2009). Alle drei sind durch eine gemeinsame Vergangenheit miteinander verbunden und pflegen gute Beziehungen in einer Art „Old Boy Network“. Asmussen und Weidmann hatten bei Weber studiert, bevor Asmussen aus seiner Position im BMF heraus darauf hinwirkte, dass Weber Bundesbankpräsident wurde, der anschließend wiederum Weidmann zu seiner Position im Bundeskanzleramt verhalf (vgl. Bohsem et al. 2008). Nicht nur während der Finanzkrise hatte diese Konstellation Sanio aus dem Netzwerk zentraler Akteure exkludiert, sondern auch vorher und nachher scheint die Person des Präsidenten für Maßnahmen, die direkte Auswirkungen auf die gesamte BaFin haben, ein zusätzlicher Auslöser gewesen zu sein. In der Debatte um einen Neuzuschnitt der Aufsichtskompetenzen zwischen BaFin und Deutscher Bundesbank, die zur Eingliederung der BaFin in die Bundesbank führen sollte (vgl. Handelsblatt 2010), hatte Sanio zwar weitgehend geschwiegen. Allerdings hatte er angekündigt, dass er sich auch als Beamter nicht „gefesselt“ fühle, sondern sich sehr wohl zu „endgültigen Entscheidungen im Gesetzgebungsverfahren “ äußern werde (Heise u. Herden 2009), was im Delegationsverhältnis zwischen BMF und BaFin einen Verstoß gegen den ausdrücklichen Willen des Prinzipals darstellt. Derartige Äußerungen wurden von Vertretern aus Politik und BMF als Drohung aufgefasst, die in eine Kategorie mit Stellungnahmen Sanios fallen, die bereits in der Vergangenheit massive Kritik nach sich gezogen hatten. Zwar wurde in keinem Fall die inhaltliche Richtigkeit der Aussagen Sanios infrage gestellt, wohl aber die Form, in der die Inhalte präsentiert worden waren. In Fragen der Aufsicht habe es intern Situationen gegeben, in denen „Sanio sowohl Weber als auch Asmussen einmal über den Mund gefahren ist und ihnen gezeigt hat, was er von ihren Fähigkeiten hält" (Interviewnachweis 12). Im Fall der HRE bezeichnete Sanio die Bank als in der „Todeszone“ (Deutscher Bundestag 2009, S. 114) befindlich und während der Griechenland-Krise sprach er von einem „Angriffskrieg gegen den Euro" (Spiegel-Online 05.05.2010). Beide Äußerungen entfalteten eine entsprechende Außenwirkung und wurden wiederum entgegen den Vorgaben der Fachaufsicht getätigt, die eine vorherige Genehmigung von öffentlichen Erklärungen durch das BMF vorsehen. In Interviews erklärte Sanio zudem, dass die BaFin personell „hoffnungslos unterbesetzt“ sei und „das Optimum [der Aufsicht] nie erreichen " könne (FTD 04.06.2009). Damit provozierte er nicht nur die Kritik der Leitung des BMF, sondern auch der Finanzpolitiker des Deutschen Bundestages. Diese brachte Sanio sowohl durch seinen Äußerungen gegen sich auf, als auch durch seine Art der Behördenleitung, die schon $2006 \mathrm{zu}$ öffentlichen Skandalen führte, als ein Beschaffungsskandal die BaFin in die Schlagzeilen brachte. Mit Plänen zur Veränderung interner Arbeitszeit- und Beurteilungssysteme hatte Sanio in der Folge Unmut unter den Mitarbeitern der BaFin herbeigeführt und diesen weiter befördert, indem er auf Konfrontationskurs mit dem Personalrat der Behörde gegangen war (vgl. Afhüppe 2006). Der Umbau der Leitungsstruktur der BaFin ist folglich einerseits als Maßnahme gegen die BaFin insgesamt zu interpretieren, andererseits als Antwort auf das Versagen Sanios als Behördenleiter. Die kollegiale Behördenleitung, in der drei Exekutivdirektoren die BaFin zusammen mit Sanio leiten, sollte demnach Alleingänge verhindern und 
gleichzeitig die Aufgabenbelastung der inneren Führung und Koordination auf andere Personen verteilen. Auch die Maßnahmen, die das Finanzministerium mit den Grundsätzen zur Rechts- und Fachaufsicht ergriffen hat, sollten nicht nur eine allzu emanzipierte Behörde „einfangen“, sondern auch einen unliebsamen Präsidenten, der als Beamter auf Lebenszeit nicht wie ein politischer Beamter (vgl. $\$ 54$ Bundesbeamtengesetz) jederzeit ohne Angabe von Gründen in den einstweiligen Ruhestand versetzt werden kann (vgl. Interviewnachweis 13).

Es liegt damit der Schluss nahe, dass das BMF Skepsis gegenüber der BaFin hegt, die von der Delegationsbeziehung beider Behörden und der besonderen Personalkonstellation herrührt. Die Ausübung der Fachaufsicht wird damit zu einer Herausforderung für das Ministerium. Darüber hinaus besteht Anlass zur Sorge um die Fähigkeit des Ministeriums, seine Kernaufgaben im Politikfeld Finanzmarktpolitik zu erfüllen. Es zeigt sich, dass Finanzmarktgesetze nicht mehr ohne Unterstützung durch private Berater erarbeitet werden können und damit die Programmvorbereitungsfunktion des BMF tangiert ist.

Das BMF kann diese Schwächen, die nicht ausschließlich innerhalb der Organisation liegen oder von dieser gesteuert werden, von sich aus nur sehr eingeschränkt beheben, sodass selbst nach der Wahrnehmung von Problemen institutionelles Lernen nicht ohne Weiteres möglich ist. Das Ministerium ist hierfür auf die Unterstützung der Politik angewiesen, die jedoch Versäumnisse und Defizite aufseiten des BMF nicht diskutiert und diese allein bei der BaFin sieht. Der Grund hierfür ist bei den Regierungsparteien zu suchen, die mit der Leitung des BMF zwischen 2005 und 2011 betraut waren. Da weder die SPD noch die CDU ein Interesse daran haben, Schwächen in der jeweiligen Amtsführung von Peer Steinbrück bzw. Wolfgang Schäuble öffentlich zu machen, bleiben Reformen der Ministerialorganisation in der Finanzmarktpolitik aus und verschwinden nach einer kurzen Phase der medialen Aufmerksamkeit wieder von der politische Agenda.

\section{Schlussbetrachtung und Ausblick}

Die eingangs aufgeworfene Frage nach der potenziellen Unfähigkeit des Finanzministeriums, einerseits die Finanzmarktpolitik gesetzgeberisch zu gestalten und andererseits seine nachgeordnete Behörde BaFin angemessen zu steuern, muss bejaht werden. Dem Ministerium drohen der Verlust der eigenen Handlungsfähigkeit aufgrund unzureichender Personalressourcen und die Zunahme der Abhängigkeit von BaFin und externen, privaten Vertragspartnern. Dieses Defizit ist zwar keineswegs die Ursache für die Finanzkrise oder Verwerfungen auf dem deutschen Finanzmarkt, es erschwerte aber den Umgang mit der Krise und verzögerte adäquate Reaktionen.

Die Krise hatte nicht nur ein „window of opportunity“ für die Anpassung von Regulierungsregimen, sondern auch für die Veränderung von Aufsichtsstrukturen - zum Beispiel die bessere Koordination zwischen BaFin und Bundesbank bei der Bankenaufsicht - und des Bereichs der Ministerialverwaltung geöffnet. Die Reformdiskussion im Anschluss an die Finanzkrise zielt daher weltweit auf die Verschärfung von Regulierung und die Effizienzsteigerung von Aufsichtsstrukturen ab. In beiden Bereichen ist in Deutschland auch das BMF betroffen, sodass die 
Frage offen bleibt, inwieweit diese Reformen auch die Handlungsfähigkeit des BMF verbessern würden.

Hinsichtlich der Veränderung von Aufsichtsstrukturen finden Prozesse statt, die auf eine Verstärkung von Aufsichtskompetenzen zugunsten der Europäischen Union hindeuten. Durch einen Beschluss des Europaparlaments wurde die Möglichkeit eröffnet, mit Beginn des Jahres 2011 europäische Aufsichtsagenturen ${ }^{6}$ für Banken-, Wertpapier- und Versicherungsaufsicht zu schaffen, die nicht nur Koordinationsaufgaben zwischen nationalen Behörden erfüllen, sondern auch begrenzte Zugriffsrechte auf nationale Marktakteure haben (vgl. Berschens 2010). Damit existieren auf europäischer Ebene neue Aufsichtsstrukturen, deren Einführung in Deutschland mit dem Verweis auf Entscheidungen der EU über viele Monate verschoben worden war (vgl. Lebert 2010). Ende 2010 gaben die Parteien der Regierungskoalition trotz der europäischen Schritte alle Reformvorhaben auf und verständigten sich auf die Beibehaltung der deutschen Finanzaufsicht mit der Aufgabentrennung zwischen Bundesbank und BaFin (Dautzenberg et al. 2010).

Der ursprünglich geplante Umbau der Finanzaufsicht, bei dem die Zuständigkeit für die Bankenaufsicht der Bundesbank übertragen (vgl. Koalitionsvertrag 2009, S. 46), die BaFin vollständig in die Bundesbank integriert (vgl. FTD 10.04.2010) oder eine Holding aus Bundesbank und BaFin (vgl. Wallstreet-Online 19.03.2010) geschaffen werden sollte, hatte das Ziel die Aufsicht in Deutschland effizienter zu gestalten. Dabei wurde in keinem Modell eine Effizienzsteigerung operationalisiert oder zumindest das Versagen des bestehenden Systems konkretisiert, sodass davon auszugehen ist, dass die Reformvorschläge keine aufsichtsrelevante Ursache hatten, sondern politisch motiviert waren. Weder in der Arbeit der BaFin noch in der Aufgabenteilung zwischen Bundesbank und BaFin wurden ernsthafte Schwächen gesehen, die eine Re-Organisation notwendig gemacht hätten (vgl. Interviewnachweis 14). Der Vorschlag, der BaFin den Kompetenzbereich der Bankenaufsicht zu entziehen, erklärt sich vielmehr aus einer polittaktischen Überlegung, die öffentlichkeitswirksam politische Handlungsfähigkeit beweisen sollte. Nach der steuerfinanzierten Rettung von Banken sahen Politiker die Notwendigkeit, steuerzahlenden Bürgern - und potenziellen Wählern - zu signalisieren, dass Bankenzusammenbrüche in Zukunft verhindert würden. Dafür sind in erster Linie regulatorische Veränderungen nötig, die aber nur mit zeitlicher Verzögerung zu realisieren und zudem schwierig in der Öffentlichkeit zu vermitteln sind. Medial leichter zu ,vermarkten' war hingegen der Vorschlag, die für Bankenaufsicht zuständige Behörde abzustrafen und die Zuständigkeit für den Bankensektor einer Behörde wie der Bundesbank zu übertragen, die in der Bevölkerung hohes Ansehen genießt (vgl. Interviewnachweis 14). Die Koalitionsvereinbarung zum Umbau der Bankenaufsicht - einschließlich einer Standortgarantie für den Sitz der BaFin in Bonn, dem Bundestagswahlkreis von FDP-Chef Guido Westerwelle (vgl. Koalitionsvertrag 2009, S. 46) - war damit ein Akt von symbo-

6 Im Januar 2011 nahmen die EBA (European Banking Authority), ESMA (European Securities and Markets Authority) und EIOPA (European Insurance and Occupational Pensions Authority) ihre Arbeit auf, die aus den ehemaligen Ebene-3-Kommittes - CEBS, CESR und CEIOPS - des sog. Lamfalussy-Verfahrens hervorgegangen sind (COM 2009). 
lischer Politik, der keine Lösung für ein institutionelles Aufsichtsproblem darstellte. Gleiches gilt für das Integrationsmodell der Bundesbank und das HoldingModell der Unionsparteien, durch die in Aufsichtsfragen jeweils ein Zugriff des Finanzministeriums auf die Bundesbank hätte erfolgen sollen. Das BMF hätte seine Steuerungsmöglichkeit dadurch noch weiter eingebüßt oder vollständig verloren, wenn gleichzeitig die Unabhängigkeit der Notenbank (vgl. Art. 88 GG; $\mathbb{1} 12$ BundesbankG) gewahrt worden wäre. Die ministeriellen Handlungsfähigkeiten in der Politikformulierung und zur Steuerung von Aufsichtsbehörden wären demnach durch kein Modell erhöht werden. Allerdings gilt dies ebenso für die Beibehaltung der bisherigen Struktur, sodass sich in Zukunft die Angst des Ministeriums vor der Aufsicht eher verstärken als abschwächen dürfte.

\section{Literatur}

Afhüppe, Sven. 2006. Sanio streitet mit Personalrat. Bundestag beschäftigt sich mit BaFinSkandal. http://www.wiwo.de/politik-weltwirtschaft/bundestag-beschaeftigt-sich-mitbafin-skandal-155594/. Zugegriffen: 19.10.2010.

Alter, Karen. 2008. Agents or Trustees? International Courts in their Political Context. European Journal of International Relations 14:33-63.

Aulich, Chris, Heba Batainah und Roger Wettenhall. 2010. Autonomy and Control in Australian Agencies. Data and Preliminary Findings from a Cross-National Empirical Study. Australian Journal of Public Administration 69:214-228.

Bach, Tobias, Julia Fleischer und Thurid Hustedt. 2010. Organisation und Steuerung zentralstaatlicher Behörden. Agenturen im westeuropäischen Vergleich. Berlin: edition sigma.

Baecker, Dirk. 2003. Organisation und Management. Frankfurt a. M.: Suhrkamp.

BaFin. 2010. Grundsätze für die Ausübung der Rechts- und Fachaufsicht des Bundesministeriums der Finanzen (BMF) über die BaFin. http://www.bafin.de/cln_152/nn_722836/ SharedDocs/Veroeffentlichungen/DE/BaFin/Grundlagen/gs_aufsicht__bmf_bafin. html. Zugegriffen: 19.10.2010.

BaFin. 2010a. Mitarbeiter. http://www.bafin.de/cln_171/nn_722838/DE/ BaFin/ Organisation/ Mitarbeiter/mitarbeiter_node.html?_nnn=true. Zugegriffen: 14.06.2011.

Barth, James R., Gerard Caprio und Ross Levine. 2004. Bank Regulation and Supervision. What Works Best? Journal of Financial Intermediation 13:205-248.

Berschens, Ruth. 2010. Parlament macht Weg für EU-Finanzaufsicht frei. Handelsblatt. 23.09.2010.

Bohsem, Guido, Helga Einecke und Ulrich Schäfer. 2008. Die sechs Nothelfer. Süddeutsche Zeitung. 13.10.2008.

Bundesministerium der Finanzen, BMF. 2010. Im Profil - Das Bundesministerium der Finanzen. Informationsbroschüre des Referats für Öffentlichkeitsarbeit.

Bundesministerium der Finanzen, BMF. 2010a. Entwurf des Bundeshaushalts 2011 und des Finanzplans 2010 bis 2014. http://www.bundesfinanzministerium.de/nn_100440/ DE/BMF__Startseite/Publikationen/Monatsbericht_des_BMF/2010/07/analysen-undberichte/b01-Bundeshaushalt-2011-und-Finanzplan-2010-bis-2014/b01-Bundeshaushalt-2011-und-Finanzplan-2010-bis-2014.html\#9. Zugegriffen: 19.10.2010. 
Bundesministerium der Finanzen, BMF. 2010b. Entwicklung der Mitarbeiteranzahl des BMF. Antwort auf ein Auskunftsersuchen nach $\mathbb{\$} 1$ IFG vom 22.10.2010.

Bundesministerium des Innern, BMI. 2008. Grundsätze zur Ausübung der Fachaufsicht der Bundesministerien über den Geschäftsbereich. http://www.verwaltung-innovativ.de/ cln_108/nn_684674/SharedDocs/Publikationen/DE/20080515_24_grundsaetze_ausuebung_fachaufsicht, templateId=raw,property=publicationFile.pdf/20080515_24_ grundsaetze_ausuebung_fachaufsicht.pdf. Zugegriffen: 19.10.2010.

Bundesrechnungshof. 2005. Bericht an den Haushaltsausschuss des Deutschen Bundestages nach $\int 88$ Abs. 2 BHO zur Fachaufsicht der Bundesministerien über ibre nachgeordneten Geschäftsbereiche. Gz. VII 4-2003-0396.

Burghof, Hans Peter. 2009. Bankenaufsicht machte eine schlechte Figur in der Krise. Betriebswirtschaftliche Blätter 12/691.

Christensen, Tom, Amund Lie und Per Lægreid. 2008. Beyond new Public Management. Agencification and Regulatory Reform in Norway. Financial Accountability \& Management 24:15-30.

Dautzenberg, Leo, Bartholomäus Kalb und Michael Meister. 2010. Koalition einig über Reform der nationalen Finanzaufsicht, Pressemitteilung der CDU/CSU, 16.12.2010. http://www.cducsu.de/Titel_pressemitteilung_koalition_einig_ueber_reform_der_nationalen_finanzaufsicht/TabID_6/SubTabID_7/InhaltTypID_1/InhaltID_17465/Inhalte.aspx. Zugegriffen: 10.01.2011.

Deutsche Bundesbank. 2010. Mitwirkung der Deutschen Bundesbank im Baseler Ausschuss für Bankenaufsicht. Antwort auf ein Auskunftsersuchen nach $\mathbb{S} 1$ IFG vom 22.09.2010.

Deutscher Bundestag. 2006. Rolle der Bundesregierung bei der Rechts- und Fachaufsicht der Bundesanstalt für Finanzdienstleistungsaufsicht (BaFin). BT-Drucksache 16/3253. 06.11.2006.

Deutscher Bundestag. 2009. Beschlussempfehlung und Bericht des 2. Untersuchungsausschusses nach Artikel 44 des Grundgesetzes (HRE-Untersuchungsausschuss). BTDrucksache 16/14000. 18.09.2009.

Deutscher Bundestag. 2009a. Mitarbeit von Privaten an Gesetzentwürfen und Arbeitsfähigkeit der Bundesministerien. Antwort der Bundesregierung auf die Kleine Anfrage der Fraktion Die Linke 16/14025. BT-Drucksache 16/14133. 26.10.2009.

Döhler, Marian. 2002. Institutional Choice and Bureaucratic Autonomy in Germany. West European Politics 25:101-124.

Döhler, Marian. 2006. Regulative Politik und die Transformation der klassischen Verwaltung. In Politik und Verwaltung. Auf dem Weg zu einer postmanagerialen Verwaltungsforschung. Politische Vierteljahresschrift Sonderheft 37, Hrsg. Jörg Bogumil, Werner Jann und Frank Nullmeier, 208-227. Wiesbaden: VS Verlag für Sozialwissenschaften.

Döhler, Marian. 2007. Vom Amt zur Agentur? Organisationsvielfalt, institutioneller Anpassungsdruck und Wandlungsprozesse im deutschen Verwaltungsmodell. Agencies in Westeuropa, Hrsg. Marian Döhler und Werner Jann, 12-47. Wiesbaden: VS Verlag für Sozialwissenschaften.

Döhler, Marian. 2007a. Die politische Steuerung der Verwaltung. Eine empirische Studie über politisch-administrative Interaktionen auf der Bundesebene. Baden-Baden: Nomos. 
Döhler, Marian. 2011. Wie Ministerien (versuchen,) Bundesbehörden (zu) steuern. Verwaltungsarchiv 102:110-133.

Downs, Anthony. 1967. Inside Bureaucracy. Boston: Little, Brown and Company.

Dunleavy, Patrick. 1991. Democracy, Bureaucracy and Public Choice. Economic Explanations in Political Science. London: Harvester.

Eberlein, Burkard. 2000. Regulierung und Konstitution von Märkten in Europa. In. Die politische Konstitution von Märkten, Hrsg. Roland Czada und Susanne Lütz, 89-106. Wiesbaden: Westdeutscher Verlag.

Ebinger, Falk, und Carina Schmitt. 2010. Alles eine Frage des Managements? Wie Autonomierechte die Handlungsfreiheit des administrativen Führungspersonals beeinflussen. Politische Vierteljahresschrift 51:69-93.

Eichhorn, Peter et al. (Hrsg.). 1985. Verwaltungslexikon. Baden-Baden: Nomos.

Financial Times Deutschland. Sanio und die leeren Gänge. 04.06.2009.

Financial Times Deutschland. Streit um Finanzaufsicht. Bundesbank will das letzte Wort haben. 10.04.2010.

Frach, Lotte. 2008. Finanzaufsicht in Deutschland und Großbritannien. Die BaFin und die FSA im Spannungsfeld der Politik. Wiesbaden: VS Verlag für Sozialwissenschaften.

Frach, Lotte. 2010. Finanzmarktregulierung in Deutschland. Baden-Baden: Nomos.

Gilardi, Fabrizio, und Dietmar Braun. 2002. Delegation aus der Sicht der Prinzipal-AgentTheorie. Ein Literaturbericht. Politische Vierteljahresschrift, 43:147-161.

Hall, Peter, und Rosemary Taylor. 1996. Political Science and the Three New Institutionalisms. Political Studies 44:936-957.

Handelsblatt. Gefährliche halbe Reform. 18.09.2007.

Handelsblatt. BaFin-Chef greift schwarz-gelbe Koalition an. 26.10.2009.

Handelsblatt. Dautzenberg will zentrale Aufsicht durch die Bundesbank. 06.10.2010.

Handelsblatt. BaFin will mehr Kontrolleure beschäftigen. 01.03.2011.

Handke, Stefan. 2010. Yes, we can (control them)! - Regulatory Agencies: Trustees or Agents? Goettingen Journal of International Law 2:111-134.

Handke, Stefan. 2010a. Ein konturloser Begriff mit Konjunktur. Das deutsche Verständnis von Finanzmarktregulierung. der moderne staat 3:53-69.

Hanke, Thomas. 2009. Outsourcing in Berlin. Die Republik der Anwälte. Handelsblatt. 28.05.2009.

Heinemann, Friedrich, und Martin Schüler. 2004. A Stiglerian view on banking supervision. Public Choice 121:99-130.

Heise, Stephanie, und Ingrid Herden. 2009. Ich fühle mich nicht gefesselt. Wirtschaftswoche. http://www.wiwo.de/finanzen/ich-fuehle-mich-nicht-gefesselt-411684/3; 24.10.2009. Zugegriffen: 19.10.2010.

Hood, Christopher, Henry Rothenstein und Robert Baldwin. 2003. The Government of Risk. Oxford: Oxford University Press.

Hulverscheidt, Claus. 2008. Gelesen und gelocht. Hypo Real Estate und die Finanzkrise. Süddeutsche Zeitung. 12.11.2008.

Kaserer, Christoph. 2006. Trends in der Bankenaufsicht als Motor der Überregulierung des Bankensektors - Anmerkungen aus einer politikökonomischen Perspektive. Perspektiven der Wirtschaftspolitik 7:67-87. 
Kessler, Oliver. 2008. Die internationale Politische Ökonomie des Risikos. Eine Analyse am Beispiel der Diskussion um die Reformierung der Finanzmärkte. Wiesbaden: VS Verlag für Sozialwissenschaften.

Kiewiet, D. Roderick, und Mathew D. McCubbins. 1991. The Logic of Delegation. Congressional Parties and the Appropriations Process. Chicago: University of Chicago Press.

Koalitionsvertrag. 2009. Wachstum, Bildung, Zusammenhalt, Koalitionsvertrag zwischen der CDU, CSU und FDP. http://www.cdu.de/doc/pdfc/091026koalitionsvertrag-cducsu-fdp.pdf. Zugegriffen: 19.10.2010.

Kurz, Andreas. 2009. Die Söldner des Rechts. Financial Times Deutschland. 12.05.2009.

Lebert, Rolf. 2010. EU-Behörden als Vorbild. Neustart der deutschen Aufsichtsreform. Financial Times Deutschland. 10.09.2010.

Luhmann, Niklas. 1994. Funktionen und Folgen formaler Organisation. Berlin: Duncker \& Humblot.

Lütz, Susanne. 2002. Der deutsche Finanzsektor im Zeichen von Europäisierung und Internationalisierung. In Entgrenzte Märkte - grenzenlose Bürokratie?, Hrsg. Volker Schneider, 144-157. Frankfurt a. M.: Campus Verlag.

Mayntz, Renate. 1978. Soziologie der öffentlichen Verwaltung. Heidelberg: C. F. Müller Verlag.

Mayntz, Renate, und Fritz W. Scharpf. 1995. Steuerung und Selbstorganisation in staatsnahen Sektoren. In Gesellschaftliche Selbstregelung und politische Steuerung, Hrsg. Renate Mayntz und Fritz W. Scharpf, 9-38. Frankfurt a. M.: Campus Verlag.

McCubbins, Mathew D., Roger G. Noll und Barry R. Weingast. 1987. Administrative Procedures as Instruments of Political Control. Journal of Law, Economics, and Organization 3:243-77.

Niskanen, William. 1979. Ein ökonomisches Modell der Bürokratie. In Ökonomische Theorie der Politik, Hrsg. Werner W. Pommerehne und Bruno S. Frey, 349-369. Berlin: Springer.

Quaglia, Lucia. 2008. Setting the Pace? Private Financial Interests and European Financial Market Integration. British Journal of Politics and International Relations 10:46-64.

Quintyn, Marc, und Michael Taylor. 2004. Should Financial Sector Regulators be independent? International Monetary Fund Papers. Economic Issues 32.

Sanio, Jochen. 2002. Die Versicherungsaufsicht nach Errichtung der BaFin. Vortrag von Jochen Sanio anlässlich der Mitgliederversammlung des Gesamtverbandes der Deutschen Versicherungswirtschaft e. V. (GDV) am 14. November 2002 in Berlin. http:// www.bafin.de/nn_992414/SharedDocs/Reden/DE/2002/re_021114__sanio.html. Zugegriffen: 19.10.2010.

Spiegel-Online. 2010. Chef-Finanzaufseher spricht von „Angriffskrieg gegen Euro-Zone“. http://www.spiegel.de/wirtschaft/unternehmen/0,1518,693239,00.html.; am 05.05.2010. Zugegriffen: 19.10.2010.

Sturm, Roland, Stephen Wilks, Markus Müller und Ian Bartle. 2002. Der regulatorische Staat. Deutschland und Großbritannien im Vergleich. London: Anglo-German Foundation.

Thatcher, Mark. 2002. Regulation After Delegation: Independent Regulatory Agencies in Europe. Journal of European Public Policy 9:954-972. 
Wallstreet-Online. 2010. Finanzaufsicht - Holding-Modell. Regierung kurz vor Einigung. http://www.wallstreet-online.de/nachrichten/nachricht/2914030-finanzaufsicht-holding-modell-regierung-kurz-vor-einigung.html. Zugegriffen: 19.10.2010.

Weiland, Severin, und John Goetz. 2009. Union rückt von Staatssekretär Asmussen ab. Spiegel Online. 25.06.2009. http:/www.spiegel.de/politik/deutschland/ 0,1518,631710,00.html. Zugegriffen: 19.10.2010.

Zitierte Interviews aus dem Jahr 2010

Anonym. Ehemaliger Mitarbeiter im Leitungsbereich der BaFin, derzeitige Beschäftigung in leitender Funktion bei einem deutschen Versicherungsunternehmen. 13.07.2010.

Binding, Lothar. Mitglied des Deutschen Bundestags, SPD-Fraktion, Mitglied des Finanzausschusses. 01.07.2010.

Dautzenberg, Leo. Mitglied des Deutschen Bundestags, CDU/CSU-Fraktion, Mitglied des Finanzausschusses. 22.06.2010.

Quinten, Daniel. Deutsche Bundesbank, Büroleiter des Vizepräsidenten Franz-Christoph Zeitler. 11.10.2010.

Sell, Michael. Bundesanstalt für Finanzdienstleistungsaufsicht, Exekutivdirektor Querschnittsaufgaben/Innere Verwaltung. 30.08.2010.

Troost, Axel. Mitglied des Deutschen Bundestags, Fraktion DIE LINKE, Mitglied des Finanzausschusses. 06.07.2010.

Wehling, Axel. Gesamtverband der Deutschen Versicherungswirtschaft, Mitglied der Hauptgeschäftsführung. 08.10.2010.

Wissing, Volker. Mitglied des Deutschen Bundestags, FDP-Fraktion, Vorsitzender des Finanzausschusses. 15.07.2010.

\section{Autorenangaben}

Dipl.-Pol. Stefan Handke,

Leibniz Universität Hannover, Institut für Politische Wissenschaft, Schneiderberg 50, 30167 Hannover,

st.handke@ipw.uni-hannover.de 